\title{
THE RIGIDITY THEOREMS OF SELF-SHRINKERS
}

\author{
QI DING AND Y. L. XIN
}

\begin{abstract}
By using certain techniques developed in minimal submanifold theory, we study the rigidity problem for self-shrinkers in the present paper. We prove rigidity results for the squared norm of the second fundamental form of self-shrinkers, either under pointwise conditions or under integral conditions.
\end{abstract}

\section{INTRODUCTION}

The subject of self-shrinkers is closely related to the theory of minimal submanifolds, as shown by previous works [5] and [6].

There is intrinsic rigidity and extrinsic rigidity for minimal submanifolds in the unit sphere. The intrinsic rigidity implies gap property of the scalar curvature, so is the squared norm of the second fundamental form by the Gauss equations. The extrinsic rigidity describes the gap phenomenon for the image of the Gauss maps. Both properties of minimal submanifolds were initiated by J. Simons in his fundamental paper 20]. Since then, extensive works have appeared to contribute to this interesting problem.

Besides the interest in its own right, the rigidity problem in the sphere is also related to the Benstein problem for minimal submanifolds in the Euclidean space 23 .

We now study the rigidity problem for self-shrinkers. Now, there is no intrinsic rigidity. However, there also exist gap phenomena for the squared norm of the second fundamental form and for the image of the Gauss maps. In the present paper, we only pay attention to the gap phenomenon for the squared norm of the second fundamental form. As for the gap phenomenon for the image under the Gauss maps, we will write another paper to contribute to the problem.

The first gap of the squared norm of the second fundamental form for selfshrinkers was obtained by Cao-Li [2] (which generalized the codimension 1 case in [15]).

Chern-do Carmo-Kobayashi in [4] confirmed that the Simons first gap in 20] is sharp and raised in order to study the subsequent gaps. Peng-Terng in [18] and [19] studied the second gap of the squared norm of the second fundamental form for compact minimal hypersurfaces in a unit sphere. They obtained pinching results for minimal hypersurfaces of constant scalar curvature in any dimension and results without the constant scalar curvature assumption in lower dimensions. After that, there are many works related to this problem. Recently, we confirmed the second gap in any dimension without constant scalar curvature assumption [7].

Received by the editors September 19, 2011.

2010 Mathematics Subject Classification. Primary 53C44.

This research was partially supported by NSFC. 
In the present paper, we employ the similar idea of our previous work 7 to study the second gap for self-shrinkers. The results will be given in Theorem 4.4 . We also study the self-shrinker surfaces in $\mathbb{R}^{3}$ with the constant squared norm of the second fundamental form. They can be classified as shown in Theorem 4.2.

By using Sobolev's inequality, Ni [17] proved gap results for minimal hypersurfaces under the integral conditions on the squared norm of the second fundamental form. For self-shrinkers, there is also Sobolev's inequality, which can be used to obtain gap results for self-shrinkers, in a manner analogous to that in [17, as shown in Theorem 3.1, But, our direct integral estimates apply to arbitrary codimension not only for self-shrinkers, but also for minimal submanifolds with corresponding modifications.

The organization of the present article is as follows: In the next section, we fix the notation and derive basic formulas as in [23, which will be used in the later sections. In $\S 3$, we prove rigidity results in higher codimension. In the final section, we give rigidity results in codimension 1.

\section{Preliminaries}

Let $M$ be an $n$-dimensional Riemannian manifold, and $X: M \rightarrow \mathbb{R}^{m+n}$ be an isometric immersion. Let $\nabla$ and $\bar{\nabla}$ be Levi-Civita connections on $M$ and $\mathbb{R}^{m+n}$, respectively. The second fundamental form $B$ is defined by $B(V, W)=\left(\bar{\nabla}_{V} W\right)^{N}=$ $\bar{\nabla}_{V} W-\nabla_{V} W$ for any vector fields $V, W$ along the submanifold $M$, where $(\cdots)^{N}$ is the projection onto the normal bundle $N M$. Similarly, $(\cdots)^{T}$ stands for the tangential projection. Taking the trace of $B$ gives the mean curvature vector $H$ of $M$ in $\mathbb{R}^{m+n}$, a cross-section of the normal bundle. In what follows we use $\nabla$ for natural connections on various bundles for notational simplicity if there is no ambiguity from the context. For $\nu \in \Gamma(N M)$, the shape operator $A^{\nu}: T M \rightarrow T M$, defined by $A^{\nu}(V)=-\left(\bar{\nabla}_{V} \nu\right)^{T}$, satisfies $\left\langle B_{V W}, \nu\right\rangle=\left\langle A^{\nu}(V), W\right\rangle$.

The second fundamental form, curvature tensors of the submanifold, curvature tensor of the normal bundle and that of the ambient manifold satisfy the Gauss equations, the Codazzi equations and the Ricci equations.

We now consider the mean curvature flow for a submanifold $M$ in $\mathbb{R}^{m+n}$. Namely, consider a one-parameter family $X_{t}=X(\cdot, t)$ of immersions $X_{t}: M \rightarrow \mathbb{R}^{m+n}$ with corresponding images $M_{t}=X_{t}(M)$ such that

$$
\left\{\begin{aligned}
\frac{d}{d t} X(x, t) & =H(x, t), \quad x \in M, \\
X(x, 0) & =X(x),
\end{aligned}\right.
$$

is satisfied, where $H(x, t)$ is the mean curvature vector of $M_{t}$ at $X(x, t)$ in $\mathbb{R}^{m+n}$.

An important class of solutions to the above mean curvature flow equations are self-similar shrinkers, whose profiles, self-shrinkers, satisfy a system of quasi-linear elliptic PDE of the second order

$$
H=-\frac{X^{N}}{2}
$$

Let $\Delta$, div and $d \mu$ be Laplacian, divergence and volume element on $M$, respectively. Colding and Minicozzi in [5] introduced a linear operator

$$
\mathcal{L}=\Delta-\frac{1}{2}\langle X, \nabla(\cdot)\rangle=e^{\frac{|X|^{2}}{4}} \operatorname{div}\left(e^{-\frac{|X|^{2}}{4}} \nabla(\cdot)\right)
$$


on self-shrinkers. They showed that $\mathcal{L}$ is self-adjoint with respect to the measure $e^{-\frac{|X|^{2}}{4}} d \mu$. In the present paper we carry out integrations with respect to this measure. We denote $\rho=e^{-\frac{|X|^{2}}{4}}$ and the volume form $d \mu$ might be omitted in the integrations for notational simplicity.

In this section we derive several basic formulas for self-shrinkers. Some of them have been known in the literature. For convenience, we include them here in our notation.

For minimal submanifolds in an arbitrary ambient Riemannian manifold J. Simons 20] derived the Laplacian of the squared norm of the second fundamental form. For arbitrary submanifolds in Euclidean space, a Simons type formula was also derived (see 21, 22], for example).

Choose a local orthonormal frame field $\left\{e_{i}, e_{\alpha}\right\}$ along $M$ with dual frame field $\left\{\omega_{i}, \omega_{\alpha}\right\}$, such that $e_{i}$ are tangent vectors of $M$ and $e_{\alpha}$ are normal to $M$. The induced Riemannian metric of $M$ is given by $d s_{M}^{2}=\sum_{i} \omega_{i}^{2}$ and the induced structure equations of $M$ are

$$
\begin{aligned}
& d \omega_{i}=\omega_{i j} \wedge \omega_{j}, \quad \omega_{i j}+\omega_{j i}=0, \\
& d \omega_{i j}=\omega_{i k} \wedge \omega_{k j}+\omega_{i \alpha} \wedge \omega_{\alpha j}, \\
& \Omega_{i j}=d \omega_{i j}-\omega_{i k} \wedge \omega_{k j}=-\frac{1}{2} R_{i j k l} \omega_{k} \wedge \omega_{l} .
\end{aligned}
$$

By Cartan's lemma we have

$$
\omega_{\alpha i}=h_{\alpha i j} \omega_{j} .
$$

Here and in the sequel we agree with the following range of indices

$$
1 \leq i, j, k \leq \cdots \leq n, \quad n+1 \leq \alpha, \beta, \gamma \leq \cdots \leq n+m .
$$

Set

$$
B_{i j}=B_{e_{i} e_{j}}=\left(\bar{\nabla}_{e_{i}} e_{j}\right)^{N}=h_{\alpha i j} e_{\alpha}, \quad S_{\alpha \beta}=h_{\alpha i j} h_{\beta i j} .
$$

Then

$$
|B|^{2}=\sum_{\alpha} S_{\alpha \alpha}
$$

From Proposition 2.2 in 22 we have

$$
\begin{aligned}
\Delta|B|^{2}=2|\nabla B|^{2}+2\left\langle\nabla_{i} \nabla_{j} H, B_{i j}\right\rangle+ & 2\left\langle B_{i j}, H\right\rangle\left\langle B_{i k}, B_{j k}\right\rangle \\
& -2 \sum_{\alpha \neq \beta}\left|\left[A^{e_{\alpha}}, A^{e_{\beta}}\right]\right|^{2}-2 \sum_{\alpha, \beta} S_{\alpha \beta}^{2} .
\end{aligned}
$$

We now suppose that the local orthonormal frame field $\left\{e_{i}\right\}_{i=1}^{n}$ is normal at a considered point $p \in M$. From the self-shrinker equations (2.1) we obtain

$$
\nabla_{j} H=\frac{1}{2}\left\langle X, e_{k}\right\rangle B_{j k}
$$

and

$$
\nabla_{i} \nabla_{j} H=\frac{1}{2} B_{i j}-\left\langle H, B_{i k}\right\rangle B_{j k}+\frac{1}{2}\left\langle X, e_{k}\right\rangle \nabla_{i} B_{j k} .
$$

Combining (2.2) and (2.4) (and using the Codazzi equation), we have

$$
\mathcal{L}|B|^{2}=2|\nabla B|^{2}+|B|^{2}-2 \sum_{\alpha \neq \beta}\left|\left[A^{e_{\alpha}}, A^{e_{\beta}}\right]\right|^{2}-2 \sum_{\alpha, \beta} S_{\alpha \beta}^{2} .
$$


This is the self-shrinker version of the well-known Simons identity. In particular, when the codimension $m=1$, the above Simons type identity reduces to the following one:

$$
\mathcal{L}|B|^{2}=2|\nabla B|^{2}+2|B|^{2}\left(\frac{1}{2}-|B|^{2}\right) .
$$

In general, we know from [20],

$$
\sum_{\alpha \neq \beta}\left|\left[A^{e_{\alpha}}, A^{e_{\beta}}\right]\right|^{2}+\sum_{\alpha, \beta} S_{\alpha \beta}^{2} \leq\left(2-\frac{1}{n}\right)|B|^{4} .
$$

When the codimension $m \geq 2$, the above estimate was refined (see [14] and [3])

$$
\sum_{\alpha \neq \beta}\left|\left[A^{e_{\alpha}}, A^{e_{\beta}}\right]\right|^{2}+\sum_{\alpha, \beta} S_{\alpha \beta}^{2} \leq \frac{3}{2}|B|^{4} .
$$

Combining (2.5) and the above inequality, we have

$$
\mathcal{L}|B|^{2} \geq 2|\nabla| B||^{2}+|B|^{2}-3|B|^{4},
$$

here we use rough estimates $|\nabla B|^{2} \geq|\nabla| B||^{2}$. It can be refined by the so-called Kato-type inequality.

From (2.4) (and using the Codazzi equation) we have

$$
\begin{aligned}
\Delta|H|^{2} & =2\left\langle H, \nabla^{2} H\right\rangle+2|\nabla H|^{2} \\
& =\left\langle H, H-2\left\langle H, B_{i k}\right\rangle B_{i k}+\left\langle X, e_{k}\right\rangle \nabla_{e_{k}} H\right\rangle+2|\nabla H|^{2} \\
& =|H|^{2}-2 \sum_{i, j}\left|\left\langle H, B_{i j}\right\rangle\right|^{2}+\frac{1}{2}\left\langle X, \nabla|H|^{2}\right\rangle+2|\nabla H|^{2} .
\end{aligned}
$$

It follows that

$$
\mathcal{L}|H|^{2}=|H|^{2}-2 \sum_{i, j}\left|\left\langle H, B_{i j}\right\rangle\right|^{2}+2|\nabla H|^{2}
$$

\section{Rigidity RESULTS IN HIGH CODIMENSION}

First of all we use formula (2.8) to obtain a rigidity result for the squared norm of the second fundamental form which was already known [2].

Proposition 3.1. Let $M^{n}$ be a complete properly immersed self-shrinker in $\mathbb{R}^{n+m}$ with $|B|^{2} \leq \frac{1}{2}$, then either $|B| \equiv 0$, and $M$ is an n-plane or $|B|^{2} \equiv \frac{1}{2}$, and $M$ is $a$ product $S^{k}(\sqrt{2 k}) \times \mathbb{R}^{n-k}$ for $1 \leq k \leq n$.

Proof. Let $\eta$ be a smooth function with compact support in $M$; then, by (2.8), we have

$$
\begin{aligned}
& \int_{M}\left(\frac{1}{2}|H|^{2}-\sum_{i, j}\left|\left\langle H, B_{i j}\right\rangle\right|^{2}+|\nabla H|^{2}\right) \eta^{2} \rho \\
= & \frac{1}{2} \int_{M}\left(\mathcal{L}|H|^{2}\right) \eta^{2} \rho=\frac{1}{2} \int_{M} \operatorname{div}\left(\rho \nabla|H|^{2}\right) \eta^{2} \\
= & -\int_{M} \eta \rho \nabla|H|^{2} \cdot \nabla \eta \leq \frac{1}{2} \int_{M}|\nabla H|^{2} \eta^{2} \rho+2 \int_{M}|H|^{2}|\nabla \eta|^{2} \rho .
\end{aligned}
$$


Since

$$
\sum_{i, j}\left|\left\langle H, B_{i j}\right\rangle\right|^{2} \leq|H|^{2}|B|^{2}
$$

we then have

$$
\int_{M}|H|^{2}\left(\frac{1}{2}-|B|^{2}\right) \eta^{2} \rho+\frac{1}{2} \int_{M}|\nabla H|^{2} \eta^{2} \rho \leq 2 \int_{M}|H|^{2}|\nabla \eta|^{2} \rho
$$

If $M$ is compact, we choose $\eta \equiv 1$, otherwise, let $\eta(X)=\eta_{r}(X)=\phi\left(\frac{|X|}{r}\right)$ for any $r>0$, where $\phi$ is a nonnegative function on $[0,+\infty)$ satisfying

$$
\phi(x)= \begin{cases}1, & \text { if } \quad x \in[0,1), \\ 0, & \text { if } \quad x \in[2,+\infty)\end{cases}
$$

and $\left|\phi^{\prime}\right| \leq C$ for some absolute constant. Noting the Euclidean volume growth of $M$ by our previous result in $\left[8\right.$ and $|H| \leq \frac{1}{2}|X|$, the right-hand side of (3.3) approaches to zero as $r \rightarrow+\infty$. This implies that $H^{2}\left(\frac{1}{2}-|B|^{2}\right) \equiv 0$ and $|\nabla H| \equiv 0$. Since $\nabla|H|^{2}=2\langle H, \nabla H\rangle$, then $|H|$ is a constant. If $|H| \equiv 0$, then $M$ is an $n$-plane. Otherwise $|H|>0$ and $|B|^{2}=\frac{1}{2}$. Moreover, (3.2) takes equality, which implies $B_{i j}=\left\langle B_{i j}, \nu\right\rangle \nu$ for any $i, j, \nu=\frac{H}{|H|}$. By Theorem 1 of Yau in [24], $M$ lies some $n+1$-dimensional linear subspace $\mathbb{R}^{n+1}$. From (2.6),$|\nabla B| \equiv 0$ which implies that the eigenvalues of $B$ are constants on $M$. In Theorem 4 of [13, Lawson showed that every smooth hypersurface with $\nabla B=0$ splits isometrically as a product of a sphere and a linear space (i.e., $S^{k} \times \mathbb{R}^{n-k}$ ). Furthermore, by the self-shrinker equation (2.1), the $k$-dimensional sphere should have the radius $\sqrt{2 k}$ and be centered at the origin.

There is a Sobolev inequality (see [16]) as follows:

$$
\kappa^{-1}\left(\int_{M} g^{\frac{2 n}{n-2}} \mathrm{~d} \mu\right)^{\frac{n-2}{n}} \leq \int_{M}|\nabla g|^{2} \mathrm{~d} \mu+\frac{1}{2} \int_{M}|H|^{2} g^{2} \mathrm{~d} \mu, \quad \forall g \in C_{c}^{\infty}(M),
$$

where $\kappa>0$ is a constant. Besides using (3.5), the Simons type inequality in self-shrinker version (2.7) would be used in the following result.

Theorem 3.1. Let $M^{n}$ be a complete immersed self-shrinker in $\mathbb{R}^{n+m}$. If $M$ satisfies an integral condition $\left(\int_{M}|B|^{n} \mathrm{~d} \mu\right)^{1 / n}<\sqrt{\frac{4}{3 n \kappa}}$, then $|B| \equiv 0$ and $M$ is a linear subspace. 
Proof. Let $\eta$ be a smooth function with compact support in $M$. Multiplying $\eta^{2}|B|^{n-2}$ on both sides of (2.7) and integrating by parts yield

$$
\begin{aligned}
0 \geq & 2 \int_{M}|\nabla| B||^{2}|B|^{n-2} \eta^{2} \rho+\int_{M}|B|^{n} \eta^{2} \rho-3 \int_{M}|B|^{n+2} \eta^{2} \rho-\int_{M} \eta^{2}|B|^{n-2} \mathcal{L}|B|^{2} \\
= & 2 \int_{M}|\nabla| B||^{2}|B|^{n-2} \eta^{2} \rho+\int_{M}|B|^{n} \eta^{2} \rho-3 \int_{M}|B|^{n+2} \eta^{2} \rho \\
& +2 \int_{M}|B| \rho \nabla|B| \cdot \nabla\left(|B|^{n-2} \eta^{2}\right) \\
= & \left.2(n-1) \int_{M}|\nabla| B\right|^{2}|B|^{n-2} \eta^{2} \rho+\int_{M}|B|^{n} \eta^{2} \rho-3 \int_{M}|B|^{n+2} \eta^{2} \rho \\
& +4 \int_{M}(\nabla|B| \cdot \nabla \eta)|B|^{n-1} \eta \rho .
\end{aligned}
$$

By the Cauchy inequality, for some $\varepsilon>0$ to be defined later, we have

$$
\begin{aligned}
3 \int_{M}|B|^{n+2} \eta^{2} \rho-\int_{M}|B|^{n} \eta^{2} \rho & +\frac{2}{\varepsilon} \int_{M}|B|^{n}|\nabla \eta|^{2} \rho \\
& \geq\left. 2(n-1-\varepsilon) \int_{M}|\nabla| B\right|^{2}|B|^{n-2} \eta^{2} \rho .
\end{aligned}
$$

Let $f=|B|^{n / 2} \rho^{1 / 2} \eta$. Integrating by parts, then we have

$$
\begin{aligned}
\int_{M}|\nabla f|^{2} & =\int_{M}\left|\nabla\left(|B|^{n / 2} \eta\right)\right|^{2} \rho+\frac{1}{2} \int_{M} \nabla\left(|B|^{n} \eta^{2}\right) \cdot \nabla \rho+\int_{M}|B|^{n} \eta^{2}\left|\nabla \rho^{1 / 2}\right|^{2} \\
& =\int_{M}\left|\nabla\left(|B|^{n / 2} \eta\right)\right|^{2} \rho-\frac{1}{2} \int_{M}|B|^{n} \eta^{2} \Delta \rho+\frac{1}{16} \int_{M}|B|^{n} \eta^{2}\left|X^{T}\right|^{2} \rho .
\end{aligned}
$$

By (2.1), we have $\Delta|X|^{2}=2 n-\left|X^{N}\right|^{2}$ (see [5] or [8]), then

$$
\Delta \rho=-\frac{\rho}{4} \Delta|X|^{2}+\left.\left.\frac{\rho}{16}|\nabla| X\right|^{2}\right|^{2}=-\frac{\rho}{4}\left(2 n-\left|X^{N}\right|^{2}\right)+\frac{\rho}{4}\left|X^{T}\right|^{2}=-\frac{n}{2} \rho+\frac{\rho}{4}|X|^{2} .
$$

From (3.8), we get (see also [9])

$$
\begin{aligned}
\int_{M}|\nabla f|^{2}= & \int_{M}\left|\nabla\left(|B|^{n / 2} \eta\right)\right|^{2} \rho-\frac{1}{8} \int_{M}|B|^{n} \eta^{2}\left|X^{N}\right|^{2} \rho \\
& +\frac{n}{4} \int_{M}|B|^{n} \eta^{2} \rho-\frac{1}{16} \int_{M}|B|^{n} \eta^{2}\left|X^{T}\right|^{2} \rho .
\end{aligned}
$$

Combining (2.1), Sobolev inequality (3.5) and (3.9), we have

$$
\begin{aligned}
& \kappa^{-1}\left(\int_{M}|f|^{\frac{2 n}{n-2}}\right)^{\frac{n-2}{n}} \leq \int_{M}|\nabla f|^{2}+\frac{1}{8} \int_{M}|B|^{n} \eta^{2}\left|X^{N}\right|^{2} \rho \\
\leq & \int_{M}\left|\nabla\left(|B|^{n / 2} \eta\right)\right|^{2} \rho+\frac{n}{4} \int_{M}|B|^{n} \eta^{2} \rho \\
= & \int_{M}\left(\frac{n^{2}}{4}|\nabla| B||^{2}|B|^{n-2} \eta^{2}+n|B|^{n-1} \eta \nabla|B| \cdot \nabla \eta+|B|^{n}|\nabla \eta|^{2}\right) \rho+\frac{n}{4} \int_{M}|B|^{n} \eta^{2} \rho .
\end{aligned}
$$


Combining the Cauchy inequality, (3.7) and (3.10), for any $\delta>0$, we have

$$
\begin{aligned}
& \kappa^{-1}\left(\int_{M}|f|^{\frac{2 n}{n-2}}\right)^{\frac{n-2}{n}} \\
\leq & (1+\delta) \frac{n^{2}}{4} \int_{M}|\nabla| B||^{2}|B|^{n-2} \eta^{2} \rho+\left(1+\frac{1}{\delta}\right) \int_{M}|B|^{n}|\nabla \eta|^{2} \rho+\frac{n}{4} \int_{M}|B|^{n} \eta^{2} \rho \\
\leq & \frac{(1+\delta) n^{2}}{8(n-1-\varepsilon)}\left(3 \int_{M}|B|^{n+2} \eta^{2} \rho-\int_{M}|B|^{n} \eta^{2} \rho+\frac{2}{\varepsilon} \int_{M}|B|^{n}|\nabla \eta|^{2} \rho\right) \\
& +\left(1+\frac{1}{\delta}\right) \int_{M}|B|^{n}|\nabla \eta|^{2} \rho+\frac{n}{4} \int_{M}|B|^{n} \eta^{2} \rho .
\end{aligned}
$$

Let $\delta=2 \frac{n-1+\varepsilon}{n}-1>0$ in (3.11), then

$$
\begin{aligned}
& \kappa^{-1}\left(\int_{M}|f|^{\frac{2 n}{n-2}}\right)^{\frac{n-2}{n}} \\
\leq & \frac{3 n}{4} \cdot \frac{n-1+\varepsilon}{n-1-\varepsilon} \int_{M}|B|^{n+2} \eta^{2} \rho+\left(\frac{n}{2 \varepsilon} \cdot \frac{n-1+\varepsilon}{n-1-\varepsilon}+1+\frac{1}{\delta}\right) \int_{M}|B|^{n}|\nabla \eta|^{2} \rho \\
\leq & \frac{3 n}{4} \cdot \frac{n-1+\varepsilon}{n-1-\varepsilon}\left(\int_{M}|B|^{2 \cdot \frac{n}{2}}\right)^{\frac{2}{n}}\left(\int_{M}\left(|B|^{n} \eta^{2} \rho\right)^{\frac{n}{n-2}}\right)^{\frac{n-2}{n}} \\
& +\left(\frac{n}{2 \varepsilon} \cdot \frac{n-1+\varepsilon}{n-1-\varepsilon}+1+\frac{1}{\delta}\right) \int_{M}|B|^{n}|\nabla \eta|^{2} \rho .
\end{aligned}
$$

Since $\left(\int_{M}|B|^{n} \mathrm{~d} \mu\right)^{1 / n}<\sqrt{\frac{4}{3 n \kappa}}$, then from (3.12) there is $0<\varepsilon_{0}<1$ such that

$$
\begin{aligned}
\kappa^{-1}\left(\int_{M}|f|^{\frac{2 n}{n-2}}\right)^{\frac{n-2}{n}} \leq & \frac{3 n}{4} \cdot \frac{n-1+\varepsilon}{n-1-\varepsilon} \cdot \frac{4\left(1-\varepsilon_{0}\right)}{3 n \kappa}\left(\int_{M}|f|^{\frac{2 n}{n-2}}\right)^{\frac{n-2}{n}} \\
& +C(\varepsilon) \int_{M}|B|^{n}|\nabla \eta|^{2} \rho,
\end{aligned}
$$

namely,

$$
\frac{(n-1+\varepsilon) \varepsilon_{0}-2 \varepsilon}{(n-1-\varepsilon) \kappa}\left(\int_{M}|f|^{\frac{2 n}{n-2}}\right)^{\frac{n-2}{n}} \leq C(\varepsilon) \int_{M}|B|^{n}|\nabla \eta|^{2} \rho .
$$

Let $\varepsilon=\frac{\varepsilon_{0}}{2}$, since $\int_{M}|B|^{n} \mathrm{~d} \mu$ is bounded, then we choose $\eta$ as Proposition 3.1 which implies $|B| \equiv 0$.

Remark. For the codimension $m=1$ case, we can use (2.6) instead of (2.7) and the pinching constant would be better.

\section{Rigidity RESUlts FOR CODIMENSION 1}

Now, we deal with the codimension $m=1$ case. We choose a local orthonormal frame field $\left\{e_{1}, \cdots, e_{n}, \nu\right\}$ in $\mathbb{R}^{n+1}$ along the hypersurface $M$ with $\left\{e_{i}\right\}_{i=1}^{n}$ tangent to $M$ and $\nu$ normal to $M$. Set the second fundamental form $B_{e_{i} e_{j}}=h_{i j} \nu$.

Define the covariant derivatives $D h$ of $h$ (with component $h_{i j k}$ ) by

$$
\sum_{k} h_{i j k} \omega_{k}=d h_{i j}-\sum_{k} h_{i k} \omega_{j k}-\sum_{k} h_{k j} \omega_{i k}
$$


and similarly we can define the covariant derivatives $h_{i j k l}$ and $h_{i j k l s}$. We have the Ricci identity

$$
\begin{aligned}
h_{i j k l}-h_{i j l k} & =\sum_{s=1}^{n} h_{i s} R_{s j k l}+\sum_{s=1}^{n} h_{s j} R_{s i k l}, \\
h_{i j k l s}-h_{i j k s l} & =\sum_{r=1}^{n} h_{r j k} R_{r i l s}+\sum_{r=1}^{n} h_{i r k} R_{r j l s}+\sum_{r=1}^{n} h_{i j r} R_{r k l s} .
\end{aligned}
$$

We need the following higher order Simons type formula for further estimates.

Theorem 4.1. Let $M^{n}$ be an immersed self-shrinker in $\mathbb{R}^{n+1}$. Then, we have

$$
\sum_{i, j, k, l} h_{i j k l}^{2}-\frac{1}{2} \mathcal{L}|\nabla B|^{2}=\left(|B|^{2}-1\right)|\nabla B|^{2}+3 \Xi+\left.\left.\frac{3}{2}|\nabla| B\right|^{2}\right|^{2},
$$

where $\Xi=\sum_{i, j, k, l, m} h_{i j k} h_{i j l} h_{k m} h_{m l}-2 \sum_{i, j, k, l, m} h_{i j k} h_{k l m} h_{i m} h_{j l}$.

Remark. At each point $p \in M, h_{i j}$ can be diagonalized $h_{i j}=\lambda_{i} \delta_{i j}$. Then,

$$
\Xi=\sum_{i, j, k} h_{i j k}^{2}\left(\lambda_{k}^{2}-2 \lambda_{i} \lambda_{j}\right)
$$

Proof. We choose a local orthonormal frame field $\left\{e_{i}\right\}_{i=1}^{n}$ and normal at a considered point $p \in M$, i.e., $\left.\nabla_{e_{i}} e_{j}\right|_{p}=0$ for any $1 \leq i, j \leq n$. By Ricci identity (4.1), we obtain

$$
\begin{aligned}
\Delta h_{i j k}= & h_{i j k l l}=\left(h_{i j l k}+h_{i r} R_{r j k l}+h_{r j} R_{r i k l}\right)_{l} \\
= & h_{i j l l k}+h_{r j l} R_{r i k l}+h_{i r l} R_{r j k l}+h_{i j r} R_{r l k l}+\left(h_{i r} R_{r j k l}+h_{r j} R_{r i k l}\right)_{l} \\
= & \left(h_{l j l i}+h_{l r} R_{r j i l}+h_{r j} R_{r l i l}\right)_{k}+h_{r j l} R_{r i k l}+h_{i r l} R_{r j k l}+h_{i j r} R_{r l k l} \\
& +h_{i r l} R_{r j k l}+h_{r j l} R_{r i k l}+h_{i r}\left(R_{r j k l}\right)_{l}+h_{r j}\left(R_{r i k l}\right)_{l} \\
= & H_{j i k}+h_{r k l} R_{r j i l}+h_{r j k} R_{r l i l}+2 h_{r j l} R_{r i k l}+2 h_{r i l} R_{r j k l}+h_{r i j} R_{r l k l} \\
& +h_{i r}\left(R_{r j k l}\right)_{l}+h_{r j}\left(R_{r i k l}\right)_{l}+h_{l r}\left(R_{r j i l}\right)_{k}+h_{r j}\left(R_{r l i l}\right)_{k} .
\end{aligned}
$$

By (2.4) (when the codimension is 1),

$$
2 H_{j i}=h_{j l i}\left\langle X, e_{l}\right\rangle+h_{i j}-2 H h_{i l} h_{j l} .
$$

Since $-\frac{1}{2}\langle X, \nu\rangle=H=\sum_{i} h_{i i}$, then

$$
\begin{aligned}
2 H_{j i k}= & h_{j l i k}\left\langle X, e_{l}\right\rangle+h_{j l i}\left\langle e_{k}, e_{l}\right\rangle+h_{j l i}\left\langle X, \bar{\nabla}_{e_{k}} e_{l}\right\rangle+h_{i j k} \\
& -2 H_{k} h_{i l} h_{j l}-2 H\left(h_{i k l} h_{j l}+h_{i l} h_{j k l}\right) \\
= & h_{j l i k}\left\langle X, e_{l}\right\rangle+2 h_{i j k}-2 H_{k} h_{i l} h_{j l}-2 H\left(h_{i l} h_{j k l}+h_{j l} h_{i k l}+h_{k l} h_{j l i}\right) .
\end{aligned}
$$

Combining (4.3) and (4.5), we obtain

$$
\begin{aligned}
\Delta h_{i j k}= & \frac{1}{2} h_{j l i k}\left\langle X, e_{l}\right\rangle+h_{i j k}-H\left(h_{i l} h_{j l k}+h_{j l} h_{i k l}+h_{k l} h_{j l i}\right)-h_{i l} h_{j l} H_{k} \\
& +h_{r k l} R_{r j i l}+h_{r j k} R_{r l i l}+2 h_{r j l} R_{r i k l}+2 h_{r i l} R_{r j k l}+h_{r i j} R_{r l k l} \\
& +h_{i r}\left(R_{r j k l}\right)_{l}+h_{r j}\left(R_{r i k l}\right)_{l}+h_{l r}\left(R_{r j i l}\right)_{k}+h_{r j}\left(R_{r l i l}\right)_{k} .
\end{aligned}
$$


A straightforward computation gives

$$
\begin{aligned}
& h_{i j k}\left(h_{r k l} R_{r j i l}+h_{r j k} R_{r l i l}+2 h_{r j l} R_{r i k l}+2 h_{r i l} R_{r j k l}+h_{r i j} R_{r l k l}\right. \\
& \left.+h_{i r}\left(R_{r j k l}\right)_{l}+h_{r j}\left(R_{r i k l}\right)_{l}+h_{l r}\left(R_{r j i l}\right)_{k}+h_{r j}\left(R_{r l i l}\right)_{k}\right) \\
= & h_{i j k}\left(6 h_{r k l} h_{r i} h_{j l}-6 h_{r k l} h_{r l} h_{i j}+3 h_{r i j} h_{r k} h_{l l}-3 h_{r i j} h_{r l} h_{k l}\right. \\
& \left.+3 h_{i r} h_{r k} h_{j l l}-2 h_{i r} h_{j k} h_{r l l}-h_{i j k} h_{r l}^{2}\right) .
\end{aligned}
$$

From Ricci identity (4.1) and (2.3), we have

$$
\begin{aligned}
\frac{h_{i j k}}{2}\left(h_{i j l k}-h_{i j k l}\right)\left\langle X, e_{l}\right\rangle & =\frac{h_{i j k}}{2}\left(h_{i r} R_{r j l k}+h_{r j} R_{r i l k}\right)\left\langle X, e_{l}\right\rangle \\
& =2 h_{i j k} h_{i r} h_{j k} H_{r}-2 h_{i j k} h_{i r} h_{r k} H_{j} .
\end{aligned}
$$

By (4.6)-(4.8), we have

$$
\begin{aligned}
& \frac{1}{2}\left(\Delta-\frac{1}{2}\langle X, \nabla \cdot\rangle\right) h_{i j k}^{2}=h_{i j k}\left(\Delta h_{i j k}-\frac{1}{2} h_{i j k l}\left\langle X, e_{l}\right\rangle\right)+h_{i j k l}^{2} \\
= & \frac{h_{i j k}}{2}\left(h_{i j l k}-h_{i j k l}\right)\left\langle X, e_{l}\right\rangle+h_{i j k}^{2}+h_{i j k l}^{2}-H h_{i j k}\left(h_{i l} h_{j l k}+h_{j l} h_{i k l}+h_{k l} h_{j l i}\right) \\
& -h_{i j k} h_{i l} h_{j l} H_{k}+h_{i j k}\left(h_{r k l} R_{r j i l}+h_{r j k} R_{r l i l}+2 h_{r j l} R_{r i k l}+2 h_{r i l} R_{r j k l}\right. \\
& \left.+h_{r i j} R_{r l k l}+h_{i r}\left(R_{r j k l}\right)_{l}+h_{r j}\left(R_{r i k l}\right)_{l}+h_{l r}\left(R_{r j i l}\right)_{k}+h_{r j}\left(R_{r l i l}\right)_{k}\right) \\
= & 2 h_{i j k} h_{i r} h_{j k} H_{r}-2 h_{i j k} h_{i r} h_{r k} H_{j}+h_{i j k}^{2}+h_{i j k l}^{2}-3 H h_{i j k} h_{i j l} h_{k l}-h_{i l} h_{j l} H_{k} h_{i j k} \\
& +h_{i j k}\left(6 h_{r k l} h_{r i} h_{j l}-6 h_{r k l} h_{r l} h_{i j}+3 h_{r i j} h_{r k} h_{l l}-3 h_{r i j} h_{r l} h_{k l}+3 h_{i r} h_{r k} h_{j l l}\right. \\
& \left.-2 h_{i r} h_{j k} h_{r l l}-h_{i j k} h_{r l}^{2}\right) \\
= & \left(1-|B|^{2}\right) h_{i j k}^{2}+h_{i j k l}^{2}+h_{i j k}\left(6 h_{i u} h_{j v} h_{u v k}-3 h_{i j u} h_{u v} h_{k v}\right)-\left.\left.\frac{3}{2}|\nabla| B\right|^{2}\right|^{2} .
\end{aligned}
$$

The quantity $\Xi$ can be estimated as follows.

For $k \neq j$, by the Cauchy inequality, we have

$$
\lambda_{k}^{2}-2 \lambda_{k} \lambda_{j} \leq \lambda_{k}^{2}+\frac{\sqrt{17}-1}{2} \lambda_{k}^{2}+\frac{\sqrt{17}+1}{2} \lambda_{j}^{2} \leq \frac{\sqrt{17}+1}{2}|B|^{2} .
$$

Then

$$
\begin{aligned}
3 \Xi \leq & \sum_{i, j, k \text { distinct }} h_{i j k}^{2}\left(\lambda_{i}^{2}+\lambda_{j}^{2}+\lambda_{k}^{2}-2\left(\lambda_{i} \lambda_{j}+\lambda_{j} \lambda_{k}+\lambda_{i} \lambda_{k}\right)\right) \\
& +3 \sum_{j, i \neq j} h_{i i j}^{2}\left(\lambda_{j}^{2}-4 \lambda_{i} \lambda_{j}\right) \\
\leq & \sum_{i, j, k \text { distinct }} h_{i j k}^{2}\left(2\left(\lambda_{i}^{2}+\lambda_{j}^{2}+\lambda_{k}^{2}\right)-\left(\lambda_{i}+\lambda_{j}+\lambda_{k}\right)^{2}\right) \\
& +3 \sum_{j, i \neq j} h_{i i j}^{2} \frac{\sqrt{17}+1}{2}|B|^{2} \\
\leq & 2|B|^{2} \sum_{i, j, k \text { distinct }} h_{i j k}^{2}+\frac{3(\sqrt{17}+1)}{2}|B|^{2} \sum_{j, i \neq j} h_{i i j}^{2} \leq \frac{\sqrt{17}+1}{2}|B|^{2}|\nabla B|^{2} .
\end{aligned}
$$


Theorem 4.2. Let $M^{2}$ be a complete proper self-shrinker in $\mathbb{R}^{3}$. If the squared norm $|B|^{2}$ of the length of the second fundamental form is a constant, then $|B|^{2} \equiv 0$ or $\frac{1}{2}$.

Proof. If mean curvature $H$ is nonpositive, then by Huisken's classification theorem (see [5], 11] and [12]) and Euclidean volume growth [8], we know the AbreschLanger curve [1] has no constant curvature, then $M$ is isometric to $S^{k} \times \mathbb{R}^{2-k}$ for $0 \leq k \leq 2$. Hence $|B|^{2} \equiv \frac{1}{2}$ or 0 .

Now, we suppose that the mean curvature $H$ changes sign and $|B|^{2}>\frac{1}{2}$. For any fixed point $p$ with mean curvature $\left.H\right|_{p}=0$, we suppose that $\left\{e_{1}, e_{2}\right\}$ is normal at the point $p$ and $h_{i j}=\lambda_{i} \delta_{i j}$ for $i=1,2$, then

$$
\lambda_{1}+\lambda_{2}=0
$$

at the point $p$. In this proof, we always carry out derivatives at $p$.

By $0=\frac{1}{2}\left(|B|^{2}\right)_{k}=\sum_{i, j} h_{i j} h_{i j k}=\lambda_{1} h_{11 k}+\lambda_{2} h_{22 k}$ and (4.11), we have

$$
h_{111}=h_{122}, \quad h_{112}=h_{222} \text {. }
$$

Then

$$
|\nabla B|^{2}=\sum_{i, j, k} h_{i j k}^{2}=4 h_{111}^{2}+4 h_{222}^{2}
$$

Since

$$
h_{11 i}+h_{22 i}=-\frac{(\langle X, \nu\rangle)_{i}}{2}=-\frac{\left\langle X, \nabla_{e_{i}} \nu\right\rangle}{2}=\frac{\left\langle X, e_{j}\right\rangle}{2} h_{i j}=\frac{\left\langle X, e_{i}\right\rangle}{2} \lambda_{i},
$$

and denote $\left\langle X, e_{i}\right\rangle$ by $x_{i}$, then by (4.12), we have

$$
h_{111}=\frac{1}{4} x_{1} \lambda_{1}, \quad h_{222}=\frac{1}{4} x_{2} \lambda_{2} .
$$

Combining (4.11), (4.13), (4.14) and $H=-\frac{\langle X, \nu\rangle}{2}=0$, we get

$$
|\nabla B|^{2}=\frac{1}{4} x_{1}^{2} \lambda_{1}^{2}+\frac{1}{4} x_{2}^{2} \lambda_{2}^{2}=\frac{1}{4}|X|^{2} \lambda_{1}^{2}=\frac{1}{8}|X|^{2}|B|^{2} .
$$

By (2.6),$|\nabla B|^{2}=|B|^{2}\left(|B|^{2}-\frac{1}{2}\right)$, we obtain

$$
|X|^{2}=8\left(|B|^{2}-\frac{1}{2}\right)=16 \lambda_{1}^{2}-4 .
$$

From the Ricci identity (4.1), we can obtain

$$
h_{i j k l}-h_{i j l k}=\left(\lambda_{i}-\lambda_{j}\right) \lambda_{i} \lambda_{j}\left(\delta_{i k} \delta_{j l}-\delta_{i l} \delta_{j k}\right) .
$$

Especially, $h_{i i k l}=h_{i i l k}$. Moreover,

$$
0=\frac{1}{2}\left(|B|^{2}\right)_{k l}=\sum_{i, j}\left(h_{i j k} h_{i j l}+h_{i j} h_{i j k l}\right) .
$$

Combining (4.4) and (4.18), we have

$$
\begin{aligned}
& h_{11 k l}=\frac{1}{4}\left(x_{1} h_{k l 1}+x_{2} h_{k l 2}+h_{k l}\right)-\frac{1}{2 \lambda_{1}} \sum_{i, j} h_{i j k} h_{i j l}, \\
& h_{22 k l}=\frac{1}{4}\left(x_{1} h_{k l 1}+x_{2} h_{k l 2}+h_{k l}\right)-\frac{1}{2 \lambda_{2}} \sum_{i, j} h_{i j k} h_{i j l} .
\end{aligned}
$$


Combining (4.11)-(4.17) and (4.19), we have

$$
\begin{aligned}
& h_{1111}=\frac{\lambda_{1}}{4}-\frac{\lambda_{1}}{8} x_{2}^{2} ; \quad h_{1122}=-\frac{\lambda_{1}}{4}-\frac{\lambda_{1}}{8} x_{2}^{2} ; \\
& h_{2211}=-\frac{\lambda_{2}}{4}-\frac{\lambda_{2}}{8} x_{1}^{2} ; \quad h_{2222}=\frac{\lambda_{2}}{4}-\frac{\lambda_{2}}{8} x_{1}^{2} ; \\
& h_{1112}=h_{1121}=\frac{1}{8} x_{1} x_{2} \lambda_{1} ; \quad h_{2212}=h_{2221}=\frac{1}{8} x_{1} x_{2} \lambda_{2} .
\end{aligned}
$$

By (4.16) and (4.20), we get

$$
\left|\nabla^{2} B\right|^{2}=\sum_{i, j, k, l} h_{i j k l}^{2}=\frac{\lambda_{1}^{2}}{16}\left(|X|^{4}+2|X|^{2}+8\right)=\lambda_{1}^{2}\left(16 \lambda_{1}^{4}-6 \lambda_{1}^{2}+1\right)
$$

and

$$
\sum_{i, j, k} h_{i j k}^{2}\left(\lambda_{k}^{2}-2 \lambda_{i} \lambda_{j}\right)=|\nabla B|^{2} \lambda_{1}^{2}
$$

By formula (4.2), we have

$$
\lambda_{1}^{2}\left(16 \lambda_{1}^{4}-6 \lambda_{1}^{2}+1\right)=2 \lambda_{1}^{2}\left(2 \lambda_{1}^{2}-\frac{1}{2}\right)\left(2 \lambda_{1}^{2}-1\right)+6 \lambda_{1}^{2}\left(2 \lambda_{1}^{2}-\frac{1}{2}\right) \lambda_{1}^{2},
$$

which implies

$$
\lambda_{1}^{2}=\frac{3}{4}
$$

By (4.16), we get

$$
|X|^{2}=8|B|^{2}-4=8 .
$$

By the formula (2.4), $\mathcal{L} H+\left(|B|^{2}-\frac{1}{2}\right) H=0$ (see also [5]), we have

$$
\mathcal{L} H+H=0 .
$$

Let the set $E=\{p \in M ; H(p)=0\}$. Since $H$ changes sign and (4.25), $E \neq \emptyset$, $\partial E=\emptyset, E \subset \partial D_{2 \sqrt{2}}$ and $H(p) \neq 0$ for any $p \in D_{2 \sqrt{2}}$. Then there is a constant $c_{1} \geq 1$ and an eigenfunction $u_{1}>0$ in some connect component $\Omega_{1}$ of $D_{2 \sqrt{2}}$ such that

$$
\left\{\begin{array}{c}
\mathcal{L} u_{1}+c_{1} u_{1}=0 \quad \text { in } \Omega_{1}, \\
\left.u_{1}\right|_{\partial \Omega_{1}}=0
\end{array}\right.
$$

Let $g=4-|X|^{2}$, then $\mathcal{L} g=-\mathcal{L}|X|^{2}=|X|^{2}-4=-g$. There is a constant $c_{2} \in(0,1]$ and an eigenfunction $u_{2}>0$ in some connect component $\Omega_{2}$ of $D_{2}$ with $\Omega_{2} \subset \Omega_{1}$ in $D_{2}$ such that

$$
\left\{\begin{array}{c}
\mathcal{L} u_{2}+c_{2} u_{2}=0 \quad \text { in } \Omega_{2} \\
\left.u_{2}\right|_{\partial \Omega_{2}}=0
\end{array}\right.
$$

By the Rayleigh quotient characterization of the first eigenvalue, we know the first eigenvalue is decreasing in domains. The above argument contradicts this fact. Therefore, the case $H$ changing sign and $|B|^{2}>\frac{1}{2}$ is impossible. 
We define the traceless part of the second fundamental form by $\stackrel{\circ}{B}=B-\frac{1}{n} g H$, where $g$ is the metric of $M$. Then we have

$$
|\stackrel{\circ}{B}|^{2}=|B|^{2}-\frac{|H|^{2}}{n} \quad \text { and } \quad|\nabla \stackrel{\circ}{B}|^{2}=|\nabla B|^{2}-\frac{|\nabla H|^{2}}{n} .
$$

In fact, by [10, the tensor $\nabla B$ could be decomposed into orthogonal components $\nabla_{i} B_{j k}=E_{i j k}+F_{i j k}$ where

$$
E_{i j k}=\frac{1}{n+2}\left(g_{i j} \nabla_{k} H+g_{i k} \nabla_{j} H+g_{j k} \nabla_{i} H\right) \quad \text { and } \quad|E|^{2}=\frac{3}{n+2}|\nabla H|^{2} .
$$

Then

$$
\begin{aligned}
|\nabla \stackrel{\circ}{B}|^{2} & =\left|\nabla\left(B-\frac{1}{n} g H\right)\right|^{2}=|\nabla B|^{2}-\frac{2}{n} \sum\left\langle B_{i j k}, \delta_{i j} H_{k}\right\rangle+\frac{1}{n}|\nabla H|^{2} \\
& =|\nabla B|^{2}-\frac{2}{3 n} \sum\left\langle B_{i j k},(n+2) E_{i j k}\right\rangle+\frac{1}{n}|\nabla H|^{2} \\
& =|\nabla B|^{2}-\frac{2(n+2)}{3 n}|E|^{2}+\frac{1}{n}|\nabla H|^{2} \\
& =|\nabla B|^{2}-\frac{2}{n}|\nabla H|^{2}+\frac{1}{n}|\nabla H|^{2}=|\nabla B|^{2}-\frac{1}{n}|\nabla H|^{2} .
\end{aligned}
$$

Theorem 4.3. Let $M^{2}$ be a complete proper self-shrinker in $\mathbb{R}^{3}$, if $|\stackrel{\circ}{B}|$ is a constant on $M$, then $|B|^{2} \equiv 0$ or $\frac{1}{2}$.

Proof. By (2.6) and (2.8), we have

$$
\mathcal{L}|\stackrel{\circ}{B}|^{2}=\mathcal{L}|B|^{2}-\frac{1}{2} \mathcal{L}|H|^{2}=2|\nabla \stackrel{\circ}{B}|^{2}+2|\stackrel{\circ}{B}|^{2}\left(\frac{1}{2}-|B|^{2}\right) .
$$

If $|\stackrel{\leftrightarrow}{B}|=0$, then $h_{i j}=\frac{H}{2} \delta_{i j}$, which implies that $M$ is an umbilical surface. So $M$ is a sphere or a plane, and we complete the proof with the self-shrinker equation. Now we suppose that $|\stackrel{\circ}{B}|$ is a positive constant, i.e., $|\stackrel{\circ}{B}|^{2}=\frac{1}{2}\left(h_{11}-h_{22}\right)^{2}+2 h_{12}^{2}>0$. Then

$$
0=\left(|\stackrel{\circ}{B}|^{2}\right)_{k}=\left(h_{11}-h_{22}\right)\left(h_{11 k}-h_{22 k}\right)+4 h_{12} h_{12 k} .
$$

Diagonalize the matrix $\left(h_{i j}\right)$ at any fixed point, then we have

$$
h_{11 k}-h_{22 k}=0,
$$

and then

$$
2|\nabla \stackrel{\circ}{B}|^{2}=|\nabla B|^{2}=|\nabla H|^{2},
$$

where the Codazzi equations have been used. Since (4.31) is independent of the choice of the frames, then it holds on the whole $M$. Now, we suppose that the mean curvature $H$ changes sign on $M$, or else we complete the proof as the argument in Theorem 4.2. Consider a point $p$ such that $H(p)=0$ and use the notation as in the proof of Theorem 4.2 then (4.11) and (4.14) still hold. Recall that $|\stackrel{B}{B}|$ is a positive constant which implies $\lambda_{1} \neq 0$. Combining (4.4), we have

$$
H_{11}=\frac{\lambda_{1}}{2}+\frac{\lambda_{1}}{8}\left(x_{1}^{2}-x_{2}^{2}\right) ; H_{22}=\frac{\lambda_{2}}{2}-\frac{\lambda_{2}}{8}\left(x_{1}^{2}-x_{2}^{2}\right) ; H_{12}=0 .
$$


Combining (4.28), (4.30), 4.31) and (4.32), we have

$$
\frac{1}{2}|\nabla H|^{2}=|\nabla \stackrel{\circ}{B}|^{2}=|\stackrel{\circ}{B}|^{2}\left(|B|^{2}-\frac{1}{2}\right)
$$

and

$H_{k} H_{k k}=\frac{1}{2}\left(|\nabla H|^{2}\right)_{k}=|\stackrel{\circ}{B}|^{2}\left(|B|^{2}\right)_{k}=2|\stackrel{\circ}{B}|^{2}\left(h_{11} h_{11 k}+h_{22} h_{22 k}\right)=0, \quad$ for $k=1,2$.

(4.33) is equivalent to

$$
x_{1}\left(\frac{1}{2}+\frac{1}{8}\left(x_{1}^{2}-x_{2}^{2}\right)\right)=0, \quad x_{2}\left(\frac{1}{2}-\frac{1}{8}\left(x_{1}^{2}-x_{2}^{2}\right)\right)=0,
$$

which implies $x_{1}=x_{2}=0$. Then, any vanishing point $p$ of the mean curvature could only be the origin, and $H$ does not change sign, which is a contradiction to our assumption.

Now, we give a result on finite integral properties about derivatives of the second fundamental form, which is useful in the later integral estimates.

Proposition 4.1. Let $M$ be a complete properly immersed self-shrinker in $\mathbb{R}^{n+1}$, if $|B|$ is bounded on $M$, then $\int_{M}\left|\nabla^{2} B\right|^{2} \rho<\infty$ and $\int_{M}|\nabla B|^{p} \rho<\infty$ for $0 \leq p \leq 4$.

Proof. Let $\eta$ be an arbitrary smooth function with compact support on $M$. By (2.6) we have

$$
\begin{aligned}
\int_{M}|\nabla B|^{2} \eta^{2} \rho & =\int_{M}|B|^{2}\left(|B|^{2}-\frac{1}{2}\right) \eta^{2} \rho+\frac{1}{2} \int_{M}\left(\mathcal{L}|B|^{2}\right) \eta^{2} \rho \\
& =\int_{M}|B|^{2}\left(|B|^{2}-\frac{1}{2}\right) \eta^{2} \rho-2 \int_{M}(\nabla|B| \cdot \nabla \eta)|B| \eta \rho \\
& \leq \int_{M}|B|^{2}\left(|B|^{2}-\frac{1}{2}\right) \eta^{2} \rho+\epsilon \int_{M}|\nabla| B||^{2} \eta^{2} \rho+\frac{1}{\epsilon} \int_{M}|B|^{2}|\nabla \eta|^{2} \rho
\end{aligned}
$$

Since $|B|$ is bounded and $M$ has Euclidean volume growth [8], then by (4.34), we get

$$
\int_{M}|\nabla B|^{2} \rho \leq \int_{M}|B|^{2}\left(|B|^{2}-\frac{1}{2}\right) \rho<\infty
$$

Using this argument for (4.2), we get

$$
\begin{aligned}
\int_{M}\left|\nabla^{2} B\right|^{2} \rho \leq & \int_{M}\left(|B|^{2}-1\right)|\nabla B|^{2} \rho+3 \int_{M} \sum_{i, j, k} h_{i j k}^{2}\left(\lambda_{k}^{2}-2 \lambda_{i} \lambda_{j}\right) \rho \\
& +\left.\left.\frac{3}{2} \int_{M}|\nabla| B\right|^{2}\right|^{2} \rho \\
< & \infty .
\end{aligned}
$$


For any $q \geq 0$, multiplying $|\nabla B|^{q} \eta^{2}$ on both sides of (2.6), and integrating by parts, we obtain

$$
\begin{aligned}
& \int_{M}|\nabla B|^{2+q} \eta^{2} \rho=\int_{M}|B|^{2}\left(|B|^{2}-\frac{1}{2}\right)|\nabla B|^{q} \eta^{2} \rho+\frac{1}{2} \int_{M}\left(\mathcal{L}|B|^{2}\right)|\nabla B|^{q} \eta^{2} \rho \\
& =\int_{M}|B|^{2}\left(|B|^{2}-\frac{1}{2}\right)|\nabla B|^{q} \eta^{2} \rho-\frac{1}{2} \int_{M} \nabla|B|^{2} \cdot \nabla\left(|\nabla B|^{q} \eta^{2}\right) \rho \\
& \leq \int_{M}|B|^{2}\left(|B|^{2}-\frac{1}{2}\right)|\nabla B|^{q} \eta^{2} \rho+q \int_{M}|B| \cdot|\nabla B|^{q}\left|\nabla^{2} B\right| \eta^{2} \rho \\
& \quad+\int_{M}|B| \cdot|\nabla B|^{q+1}\left|\nabla \eta^{2}\right| \rho \\
& \leq C \int_{M}|\nabla B|^{q} \eta^{2} \rho+\frac{1}{2} \int_{M}|\nabla B|^{2 q} \eta^{2} \rho+C \int_{M}\left|\nabla^{2} B\right|^{2} \eta^{2} \rho+C \int_{M}|\nabla B|^{q+1}\left|\nabla \eta^{2}\right| \rho,
\end{aligned}
$$

where we have used Young's inequality in the last inequality of (4.37). By (4.35) and (4.36), we know $\int_{M}|\nabla B|^{3} \rho<\infty$ for $q=1$ in (4.37) and $\int_{M}|\nabla B|^{4} \rho<\infty$ for $q=2$ in (4.37). By the Hölder inequality, we get this proposition.

In what follows, we always denote $S=|B|^{2}$. Define

$$
f=\sum_{i, j}\left(\lambda_{i}-\lambda_{j}\right)^{2} \lambda_{i}^{2} \lambda_{j}^{2}, \quad f_{3}=\sum_{i} \lambda_{i}^{3}, \quad f_{4}=\sum_{i} \lambda_{i}^{4},
$$

where $h_{i j}=\lambda_{i} \delta_{i j}$ at the considered point. Then

$$
f=2\left(S f_{4}-f_{3}^{2}\right) .
$$

It is a higher order invariant of the second fundamental form.

Lemma 4.1.

$$
\int_{M} \Xi \rho=\frac{1}{2} \int_{M} f \rho-\frac{1}{4} \int_{M}|\nabla S|^{2} \rho .
$$

Proof. By the Stokes formula, we have

$$
-\int_{M} \sum_{i, j}\left(h_{i j} \rho\right)_{j}\left(f_{3}\right)_{i}=\int_{M} \sum_{i, j} h_{i j}\left(f_{3}\right)_{i j} \rho .
$$

Since $H=-\frac{\langle X, \nu\rangle}{2}$, then

$$
\sum_{j}\left(h_{i j} \rho\right)_{j}=\sum_{j} h_{j j i} \rho-\sum_{j} h_{i j} \frac{\left\langle X, e_{j}\right\rangle}{2} \rho=-\frac{e_{i}\langle X, \nu\rangle}{2} \rho+\frac{\left\langle X, \nabla_{e_{i}} \nu\right\rangle}{2} \rho=0
$$

and combining the Ricci identity (4.1)

$$
t_{i j}=h_{i j i j}-h_{j i j i}=\lambda_{i} \lambda_{j}\left(\lambda_{i}-\lambda_{j}\right) \quad \forall i, j,
$$

we have

$$
\begin{aligned}
\left(f_{3}\right)_{l l}=\sum_{i, j, k}\left(3 h_{i j l} h_{j k} h_{k i}\right)_{l}=3 \sum_{i, j, k}\left(h_{i j l l} h_{j k} h_{i k}+h_{i j l} h_{k j l} h_{k i}+h_{i j l} h_{j k} h_{i k l}\right) \\
=3 \sum_{i} h_{i i l l} \lambda_{i}^{2}+6 \sum_{i, j} h_{i j l}^{2} \lambda_{i}=3 \sum_{i}\left(h_{l l i i} \lambda_{i}^{2}+\lambda_{i}^{3} \lambda_{l}\left(\lambda_{i}-\lambda_{l}\right)\right)+6 \sum_{i, j} h_{i j l}^{2} \lambda_{i} .
\end{aligned}
$$


Combining (4.38)-4.40), we get

$$
\begin{aligned}
0= & \int_{M} \sum_{i, l}\left(h_{l l i i} \lambda_{i}^{2} \lambda_{l}+\lambda_{i}^{3} \lambda_{l}^{2}\left(\lambda_{i}-\lambda_{l}\right)\right) \rho+2 \int_{M} \sum_{i, j, l} h_{i j l}^{2} \lambda_{i} \lambda_{l} \rho \\
= & \frac{1}{2} \int_{M} \sum_{i} \lambda_{i}^{2} S_{i i} \rho-\int_{M} \sum_{i, j, k} \lambda_{i}^{2} h_{j k i}^{2} \rho \\
& +\int_{M} \sum_{i, l} \lambda_{i}^{3} \lambda_{l}^{2}\left(\lambda_{i}-\lambda_{l}\right) \rho+2 \int_{M} \sum_{i, j, l} h_{i j l}^{2} \lambda_{i} \lambda_{l} \rho \\
= & \frac{1}{2} \int_{M} \sum_{i, j, k} h_{i k} h_{j k} S_{i j} \rho-\int_{M} \Xi \rho+\frac{1}{2} \int_{M} f \rho .
\end{aligned}
$$

By the Stokes formula and (4.39),

$$
\begin{aligned}
\int_{M} \sum_{i, j, k} h_{i k} h_{j k} S_{i j} \rho & =-\int_{M} \sum_{i, j, k}\left(h_{i k} h_{j k} \rho\right)_{j} S_{i} \\
& =-\int_{M} \sum_{i, j, k} h_{i j k} h_{j k} S_{i} \rho=-\frac{1}{2} \int_{M}|\nabla S|^{2} \rho .
\end{aligned}
$$

Combining (4.41) and (4.42), we complete the proof.

Lemma 4.2. Keeping notation as above, then $3 \Xi \leq\left(S+C_{1} f^{1 / 3}\right)|\nabla B|^{2}$; here $C_{1}=\frac{2 \sqrt{6}+3}{\sqrt[3]{21 \sqrt{6}+103 / 2}}$.

Proof. For any three distinct positive integers $i, j, k \in\{1, \cdots, n\}$, if $\lambda_{i} \lambda_{j} \leq 0$ and $\lambda_{i} \lambda_{k} \leq 0$, then by the Cauchy inequality, $2\left|\lambda_{i} \lambda_{j}\right| \leq \frac{1}{2}\left(\lambda_{i}-\lambda_{j}\right)^{2}$ which implies $\left|\lambda_{i} \lambda_{j}\right|^{3} \leq \frac{1}{4}\left(\lambda_{i}-\lambda_{j}\right)^{2} \lambda_{i}^{2} \lambda_{j}^{2}$, and

$$
\left(\left|\lambda_{i} \lambda_{j}\right|+\left|\lambda_{i} \lambda_{k}\right|\right)^{3} \leq 4\left(\left|\lambda_{i} \lambda_{j}\right|^{3}+\left|\lambda_{i} \lambda_{k}\right|^{3}\right) \leq\left(\lambda_{i}-\lambda_{j}\right)^{2} \lambda_{i}^{2} \lambda_{j}^{2}+\left(\lambda_{i}-\lambda_{k}\right)^{2} \lambda_{i}^{2} \lambda_{k}^{2} \leq \frac{f}{2} .
$$

Since there must be a nonnegative number in three numbers $\left\{\lambda_{i} \lambda_{j}, \lambda_{j} \lambda_{k}, \lambda_{i} \lambda_{k}\right\}$, we always have

$$
-\left(\lambda_{i} \lambda_{j}+\lambda_{j} \lambda_{k}+\lambda_{i} \lambda_{k}\right) \leq\left(\frac{f}{2}\right)^{1 / 3} .
$$

On the other hand, by a simple computation, the function

$$
\zeta(x) \triangleq x^{2}(1+x)^{2}(4 x-1)^{-3}
$$

on $\left(\frac{1}{4},+\infty\right)$ attains its minimum at $x=1+\sqrt{\frac{3}{2}}$. If $\lambda_{j}=-x \lambda_{i}$, then

$$
\begin{aligned}
\left(-\lambda_{i}^{2}-4 \lambda_{i} \lambda_{j}\right)^{3} & =(4 x-1)^{3} \lambda_{i}^{6} \leq \frac{x^{2}(1+x)^{2}}{\zeta(1+\sqrt{1.5})} \lambda_{i}^{6} \\
& =\frac{1}{\zeta(1+\sqrt{1.5})}\left(\lambda_{i}-\lambda_{j}\right)^{2} \lambda_{i}^{2} \lambda_{j}^{2} \leq \frac{f}{2 \zeta(1+\sqrt{1.5})} .
\end{aligned}
$$


Let $C_{1}=\frac{2 \sqrt{6}+3}{\sqrt[3]{21 \sqrt{6}+51.5}}$, by the definition of $\Xi$ and (4.43), (4.44), we have

$$
\begin{aligned}
3 \Xi \leq & \sum_{i, j, k \text { distinct }} h_{i j k}^{2}\left(\lambda_{i}^{2}+\lambda_{j}^{2}+\lambda_{k}^{2}-2\left(\lambda_{i} \lambda_{j}+\lambda_{j} \lambda_{k}+\lambda_{i} \lambda_{k}\right)\right) \\
& +3 \sum_{j, i \neq j} h_{i i j}^{2}\left(\lambda_{j}^{2}-4 \lambda_{i} \lambda_{j}\right) \\
\leq & \sum_{i, j, k \text { distinct }} h_{i j k}^{2}\left(S+\sqrt[3]{4} f^{1 / 3}\right)+3 \sum_{j, i \neq j} h_{i i j}^{2}\left(\lambda_{i}^{2}+\lambda_{j}^{2}+\sqrt[3]{\frac{f}{2 \zeta(1+\sqrt{1.5})}}\right) \\
\leq & \left(S+C_{1} f^{1 / 3}\right)|\nabla B|^{2} .
\end{aligned}
$$

By the previous definition of $t_{i j}$,

$$
\begin{aligned}
\sum_{i, j, k, l} h_{i j k l}^{2} & \geq 3 \sum_{i \neq j} h_{i j i j}^{2}=3 \sum_{i<j}\left(h_{i j i j}^{2}+\left(h_{i j i j}-t_{i j}\right)^{2}\right) \\
& =3 \sum_{i \neq j}\left(h_{i j i j}-\frac{t_{i j}}{2}\right)^{2}+\frac{3}{4} \sum_{i \neq j} t_{i j}^{2} \geq \frac{3}{4} \sum_{i \neq j}\left(\lambda_{i}-\lambda_{j}\right)^{2} \lambda_{i}^{2} \lambda_{j}^{2} .
\end{aligned}
$$

Now, we are in a position to prove a second gap property for self-shrinkers.

Theorem 4.4. Suppose that $M^{n}$ is a complete properly immersed self-shrinker in $\mathbb{R}^{n+1}$. If there exists a positive number $\delta=0.011$ such that $\frac{1}{2} \leq|B|^{2} \leq \frac{1}{2}+\delta$, then $|B|^{2} \equiv \frac{1}{2}$.

Proof. By (4.2), (4.45) and Lemmas 4.1 and 4.2, for some fixed $0<\theta<1$ to be defined later, we have

$$
\begin{aligned}
& \frac{3}{4}(1-\theta) \int_{M} f \rho+\frac{3}{8} \theta \int_{M}|\nabla S|^{2} \rho \\
= & \frac{3}{4} \int_{M} f \rho-\frac{3}{2} \theta \int_{M} \Xi \rho \leq \int_{M}\left|\nabla^{2} B\right|^{2} \rho-\frac{3}{2} \theta \int_{M} \Xi \rho \\
= & \int_{M}(S-1)|\nabla B|^{2} \rho+3\left(1-\frac{\theta}{2}\right) \int_{M} \Xi \rho+\frac{3}{2} \int_{M}|\nabla S|^{2} \rho \\
\leq & \int_{M}(S-1)|\nabla B|^{2} \rho+\left(1-\frac{\theta}{2}\right) \int_{M}\left(S+C_{1} f^{1 / 3}\right)|\nabla B|^{2} \rho+\frac{3}{2} \int_{M}|\nabla S|^{2} \rho \\
\leq & \int_{M}\left(\left(2-\frac{\theta}{2}\right) S-1\right)|\nabla B|^{2} \rho+\frac{3}{2} \int_{M}|\nabla S|^{2} \rho+\frac{3}{4}(1-\theta) \int_{M} f \rho \\
& +\frac{4}{9} C_{1}^{\frac{3}{2}}\left(1-\frac{\theta}{2}\right)^{\frac{3}{2}}(1-\theta)^{-\frac{1}{2}} \int_{M}|\nabla B|^{3} \rho,
\end{aligned}
$$

where we have used Young's inequality in the last step of the above inequality, then (4.47)

$$
0 \leq \int_{M}\left(\left(2-\frac{\theta}{2}\right) S-1\right)|\nabla B|^{2} \rho+\left(\frac{3}{2}-\frac{3 \theta}{8}\right) \int_{M}|\nabla S|^{2} \rho+C_{2}(n, \theta) \int_{M}|\nabla B|^{3} \rho,
$$

where $C_{2}=C_{2}(n, \theta)=\frac{4}{9} C_{1}^{\frac{3}{2}}\left(1-\frac{\theta}{2}\right)^{\frac{3}{2}}(1-\theta)^{-\frac{1}{2}}$. 
By (2.6), for some $\epsilon>0$ to be defined later, we have

$$
\begin{aligned}
\int_{M}|\nabla B|^{3} \rho & =\int_{M} S\left(S-\frac{1}{2}\right)|\nabla B| \rho+\frac{1}{2} \int_{M}(|\nabla B| \mathcal{L} S) \rho \\
& =\int_{M} S\left(S-\frac{1}{2}\right)|\nabla B| \rho-\frac{1}{2} \int_{M}(\nabla|\nabla B| \cdot \nabla S) \rho \\
& \leq \int_{M} S\left(S-\frac{1}{2}\right)|\nabla B| \rho+\epsilon \int_{M}\left|\nabla^{2} B\right|^{2} \rho+\frac{1}{16 \epsilon} \int_{M}|\nabla S|^{2} \rho .
\end{aligned}
$$

Combining (4.2) and (4.10), we obtain

$$
\int_{M}\left|\nabla^{2} B\right|^{2} \rho \leq \int_{M}\left(\frac{\sqrt{17}+3}{2} S-1\right)|\nabla B|^{2} \rho+\frac{3}{2} \int_{M}|\nabla S|^{2} \rho,
$$

with the help of the above inequality, (4.48) becomes

$$
\begin{aligned}
\int_{M}|\nabla B|^{3} \rho \leq & \int_{M} S\left(S-\frac{1}{2}\right)|\nabla B| \rho+\epsilon \int_{M}\left(\frac{\sqrt{17}+3}{2} S-1\right)|\nabla B|^{2} \rho \\
& +\left(\frac{3 \epsilon}{2}+\frac{1}{16 \epsilon}\right) \int_{M}|\nabla S|^{2} \rho .
\end{aligned}
$$

Multiplying $S$ on both sides of (2.6), and integrating by parts, we see

$$
\begin{aligned}
\frac{1}{2} \int_{M}|\nabla S|^{2} \rho & =\int_{M} S^{2}\left(S-\frac{1}{2}\right) \rho-\int_{M} S|\nabla B|^{2} \rho \\
& =\int_{M} S\left(S-\frac{1}{2}\right)^{2} \rho+\frac{1}{2} \int_{M} S\left(S-\frac{1}{2}\right) \rho-\int_{M} S|\nabla B|^{2} \rho \\
& =\int_{M}\left(\frac{1}{2}-S\right)|\nabla B|^{2} \rho+\int_{M} S\left(S-\frac{1}{2}\right)^{2} \rho .
\end{aligned}
$$

Combining (4.47), (4.49) and (4.50), we get

$$
\begin{aligned}
0 \leq & \int_{M}\left(\left(2-\frac{\theta}{2}\right) S-1+C_{2} \epsilon\left(\frac{\sqrt{17}+3}{2} S-1\right)\right)|\nabla B|^{2} \rho+C_{2} \int_{M} S\left(S-\frac{1}{2}\right)|\nabla B| \rho \\
& +\left(\frac{3}{2}-\frac{3 \theta}{8}+C_{2}\left(\frac{3 \epsilon}{2}+\frac{1}{16 \epsilon}\right)\right) \int_{M}|\nabla S|^{2} \rho \\
= & \int_{M}\left(\left(2-\frac{\theta}{2}\right) S-1+C_{2} \epsilon\left(\frac{\sqrt{17}+3}{2} S-1\right)\right)|\nabla B|^{2} \rho+C_{2} \int_{M} S\left(S-\frac{1}{2}\right)|\nabla B| \rho \\
& +\left(3-\frac{3 \theta}{4}+C_{2}\left(3 \epsilon+\frac{1}{8 \epsilon}\right)\right)\left(\int_{M}\left(\frac{1}{2}-S\right)|\nabla B|^{2} \rho+\int_{M} S\left(S-\frac{1}{2}\right)^{2} \rho\right) \\
= & \int_{M}\left(-\frac{\theta}{4}+\frac{\sqrt{17}-1}{4} C_{2} \epsilon-\left(1-\frac{\theta}{4}-\frac{\sqrt{17}-3}{2} C_{2} \epsilon+\frac{C_{2}}{8 \epsilon}\right)\left(S-\frac{1}{2}\right)\right)|\nabla B|^{2} \rho \\
& +C_{2} \int_{M} S\left(S-\frac{1}{2}\right)|\nabla B| \rho+\left(3-\frac{3 \theta}{4}+C_{2}\left(3 \epsilon+\frac{1}{8 \epsilon}\right)\right) \int_{M} S\left(S-\frac{1}{2}\right)^{2} \rho .
\end{aligned}
$$


By the Cauchy-Schwartz inequality and (2.6), we have

$$
\begin{aligned}
& \int_{M} S\left(S-\frac{1}{2}\right)|\nabla B| \rho \\
\leq & 2\left(\frac{1}{2}+\delta\right) \epsilon \int_{M} S\left(S-\frac{1}{2}\right) \rho+\frac{1}{8(1 / 2+\delta) \epsilon} \int_{M} S\left(S-\frac{1}{2}\right)|\nabla B|^{2} \rho \\
= & \int_{M}\left((1+2 \delta) \epsilon+\frac{S(S-1 / 2)}{8(1 / 2+\delta) \epsilon}\right)|\nabla B|^{2} \rho \\
\leq & \int_{M}\left((1+2 \delta) \epsilon+\frac{S-1 / 2}{8 \epsilon}\right)|\nabla B|^{2} \rho .
\end{aligned}
$$

Combining (2.6), (4.51) and (4.52), we have

$$
\begin{aligned}
0 \leq & \int_{M}\left(-\frac{\theta}{4}+\frac{\sqrt{17}-1}{4} C_{2} \epsilon-\left(1-\frac{\theta}{4}-\frac{\sqrt{17}-3}{2} C_{2} \epsilon+\frac{C_{2}}{8 \epsilon}\right)\left(S-\frac{1}{2}\right)\right)|\nabla B|^{2} \rho \\
& +C_{2} \int_{M}\left((1+2 \delta) \epsilon+\frac{S-1 / 2}{8 \epsilon}\right)|\nabla B|^{2} \rho \\
& +\left(3-\frac{3 \theta}{4}+C_{2}\left(3 \epsilon+\frac{1}{8 \epsilon}\right)\right) \delta \int_{M} S\left(S-\frac{1}{2}\right) \rho \\
= & \int_{M}\left(-\frac{\theta}{4}+C_{2} \epsilon\left(\frac{\sqrt{17}+3}{4}+5 \delta\right)+\left(3-\frac{3}{4} \theta\right) \delta+\frac{C_{2} \delta}{8 \epsilon}\right)|\nabla B|^{2} \rho \\
& -\int_{M}\left(1-\frac{\theta}{4}-\frac{\sqrt{17}-3}{2} C_{2} \epsilon\right)\left(S-\frac{1}{2}\right)|\nabla B|^{2} \rho .
\end{aligned}
$$

Let $\epsilon=\sqrt{\frac{\delta}{2(\sqrt{17}+3)+40 \delta}}, \theta=1 / 2$, then $C_{2}=\frac{\sqrt{6}}{6} C_{1}^{\frac{3}{2}} \leq 0.8933$, and $\frac{7}{8}-\frac{\sqrt{17}-3}{2} C_{2} \epsilon>0$,

$$
0 \leq\left(-\frac{1}{8}+\frac{0.8933}{\sqrt{2}} \sqrt{\delta\left(\frac{\sqrt{17}+3}{4}+5 \delta\right)}+\frac{21}{8} \delta\right) \int_{M}|\nabla B|^{2} \rho .
$$

If we choose $\delta=0.011$, then $|\nabla B| \equiv 0$.

\section{REFERENCES}

1. U. Abresch and J. Langer, The normalized curve shortening flow and homothetic solutions, J. Differential Geometry. 23 (1986), 175-196. MR845704 (88d:53001)

2. Huai-Dong Cao and Haizhong Li, A Gap Theorem for Self-shrinkers of the Mean Curvature Flow in Arbitrary Codimension, Calc. Var. Partial Differential Equations 46 (2013), no. 3-4, 879-889. MR3018176

3. Qing Chen and Senlin Xu, Rigidity of compact minimal submanifolds in a unit sphere, Geom. Dedicata, 45(1) (1993), 83-88. MR 1199729 (93k:53057)

4. S. S. Chern, M. do Carmo, and S. Kobayashi: Minimal submanifolds of constant length, Functional Analysis and Related Fields (F. E. Browder, ed.), Springer, New York (1970). MR0273546 (42:8424)

5. Tobias H. Colding and William P. Minicozzi II, Generic Mean Curvature Flow I; Generic Singularities, Ann. of Math. (2) 175 (2012), no. 2, 755-833. MR2993752

6. Tobias H. Colding and William P. Minicozzi, II, Smooth Compactness of Self-shrinkers, Comment. Math. Helv. 87 (2012), no. 2, 463-475. MR2914856

7. Qi Ding and Y.L. Xin, On Chern's problem for rigidity of minimal hypersurfaces in the spheres, Adv. Math. 227 (2011), 131-145. MR2782189(2012b:53067)

8. Qi Ding and Y.L. Xin, Volume growth, eigenvalue and compactness for self-shrinkers, Asian J. Math. 17 (2013), no. 3, 443-456. MR.3119795 
9. Klaus Ecker, Logarithmic Sobolev inequalities on submanifolds of Euclidean space, J. reine angew. Math., 522 (2000), 105-118. MR1758578 (2001h:58035)

10. Gerhard Huisken, Flow by mean curvature of convex surfaces into spheres, J. Differential Geom., 20 (1984), no. 1, 237-266. MR772132 (86j:53097)

11. Gerhard Huisken, Asymptotic Behavior for Singularities of the Mean Curvature Flow, J. Differential Geom., 31 (1990), 285-299. MR1030675 (90m:53016)

12. Gerhard Huisken, Local and global behaviour of hypersurfaces moving by mean curvature. Differential geometry: partial differential equations on manifolds (Los Angeles, CA, 1990), Proc. Sympos. Pure Math., 54, Part 1, Amer. Math. Soc., Providence, RI, (1993), 175-191. MR1216584 (94c:58037)

13. H.B. Lawson, Jr., Local rigidity theorems for minimal hypersurfaces, Ann. of Math., 89(2) (1969), 187-197. MR0238229 (38:6505)

14. An-min Li and Jimin Li, An Intrinsic Rigidity Theorem for Minimal Submanifolds in a Sphere, Arch. Math., 58 (1992), 582-594. MR1161925 (93b:53050)

15. Nam Q. Le and N. Sesum, Blow-up rate of the mean curvature during the mean curvature flow and a gap theorem for self-shrinkers, Comm. Anal. Geom. 19 (2011), no. 4, 633-659. MR2880211

16. J. Michael and L.M. Simon, Sobolev and mean-value inequalities on generalized submanifolds of $\mathbb{R}^{n}$, Comm. Pure Appl. Math., 26 (1973), 361-379. MR0344978 (49:9717)

17. Lei Ni, Gap theorems for minimal submanifolds in $\mathbb{R}^{n+1}$, Comm. Analy. Geom., 9(3) (2001), 641-656. MR1895136 (2002m:53097)

18. C.K. Peng and C.L. Terng, Minimal hypersurfaces of sphere with constant scalar curvature, Ann. of Math. Stud. 103 (1983), 177-198. MR795235 (87k:53143)

19. C.K. Peng and C.L. Terng, The scalar curvature of minimal hypersurfaces in spheres, Math. Ann. 266(1) (1983), 105-113. MR722930 (85c:53099)

20. J. Simons, Minimal varieties in Riemannian manifolds, Ann. Math. 88 (1968), 62-105. MR0233295 (38:1617)

21. Knut Smoczyk, Self-Shrinkers of the Mean Curvature Flow in Arbitrary Codimension, International Mathematics Research Notices, 2005(48), (2005), 2983-3004. MR 2189784 (2006j:53098)

22. Y.L. Xin, Mean Curvature Flow With Convex Gauss Image, Chinese Annals of MathematicsSeries B, 29(2), (2008), 121-134. MR2392328 (2009b:53111)

23. Y. L. Xin, Minimal Submanifolds and Related Topics, World Scientific Publ., (2003). MR2035469 (2004m:53112)

24. S.T. Yau, Submanifolds with constant mean curvature, Amer. J. Math., 96(2) (1974), 346366. MR0370443(51:6670)

Institute of Mathematics, Fudan University, Shanghai 200433, People's Republic of CHINA

E-mail address: 09110180013@fudan.edu.cn

Institute of Mathematics, Fudan University, Shanghai 200433, People's Republic of China

E-mail address: ylxin@fudan.edu.cn 